\title{
Novel caged clusters of silicon: Fullerenes, Frank-Kasper polyhedron and cubic
}

\author{
VIJAY KUMAR \\ Institute for Materials Research, Tohoku University, 2-1-1 Katahira, Aoba-ku, Sendai 980-8577, Japan and \\ Dr Vijay Kumar Foundation, 45 Bazaar Street, K. K. Nagar (West), Chennai 600 078, India
}

\begin{abstract}
We review recent findings of metal (M) encapsulated caged clusters of $\mathrm{Si}$ and Ge obtained from computer experiments based on an ab initio pseudopotential method. It is shown that one $M$ atom changes drastically the properties of $\mathrm{Si}$ and Ge clusters and that depending upon the size of the $\mathrm{M}$ atom, cages of 14, 15, and $16 \mathrm{Si}$ as well as $\mathrm{Ge}$ atoms are formed. In particular $\mathrm{M} @ \mathrm{Si}_{16}$ silicon fullerene has been obtained for $M=Z r$ and Hf, while a Frank-Kasper polyhedron has been obtained for $M @ X_{16}, X=S i$ and Ge. These clusters show high stability and large highest occupied-lowest unoccupied molecular orbital (HOMO-LUMO) gaps which are likely to make these species strongly abundant. A regular icosahedral $\mathrm{M} @ \mathrm{X}_{12}$ cluster has also been obtained for $X=G e$ and $S n$ by doping a divalent $M$ atom. Interactions between clusters are rather weak. This is attractive for developing self-assembled cluster materials.
\end{abstract}

Keywords. Silicon; fullerenes; Frank-Kasper polyhedron; cubic.

\section{Introduction}

Silicon is the most important material for device applications. The continued shrinking size of devices has created great interest in understanding the properties of its nanoforms in order to find components of the future miniature devices. Extensive efforts have been devoted in recent years (Kumar et al 2002; Kumar and Kawazoe, to be published) to understand the properties of $\mathrm{Si}$ clusters and to find ways to produce novel nanostructures for miniature devices. The finding of visible luminescence in silicon nanoclusters is interesting for applications in optoelectronics, tunable lasers, tagging, displays etc. While the small clusters of $\mathrm{Si}$ tend to have close packed structures, clusters with 14-25 atoms have prolate structures in which $\mathrm{Si}_{9}$ or $\mathrm{Si}_{10}$ is the building block (Ho et al 1998; Mitas et al 2000). Beyond this size, compact 3-D structures are believed to be more favoured. Figure 1 shows the lowest energy structures of $\mathrm{Si}_{9}, \mathrm{Si}_{10}$ and $\mathrm{Si}_{20}$. Studies (Röthlisberger et al 1994) on $\mathrm{Si}_{45}$ do show a fullerenelike $(f)$ structure with a core of silicon atoms.

Recent experiments (Hiura et al 2001) on reactions of silane gas with transition metals showed particular stability of $\mathrm{Si}_{12} \mathrm{~W}$ cluster that did not adsorb any hydrogen. It has a hexagonal $(h)$ prism structure with $\mathrm{W}$ at the centre (figure 1). This structure is very different from the structures of elemental Si clusters. In another experiment (Beck 1989), coevaporation of $\mathrm{Si}$ and $\mathrm{M}$ led to large abundances of $\mathrm{Si}_{15} \mathrm{M}$ and $\mathrm{Si}_{16} \mathrm{M}$ clusters with $\mathrm{M}=\mathrm{Cr}$, $\mathrm{Mo}$, and $\mathrm{W}$ and little intensities for $\mathrm{Si}_{12} \mathrm{M}$ contrary to the results obtained with $\mathrm{SiH}_{4}$. Also these results are markedly different from the abundance spectrum (Jarrold and
Bower 1988) of pure $\mathrm{Si}$ clusters which shows less intensities in the region of $12-16$ atoms. Jackson and Nellermoe (1996) attempted to stabilize a dodecahedral $\mathrm{Si}_{20}$ cage with a $\mathrm{Zr}$ atom at the centre (figure 2(a)) and obtained a large binding energy (BE). However, studies by Kumar and Kawazoe (2001) showed distortions in this (a)
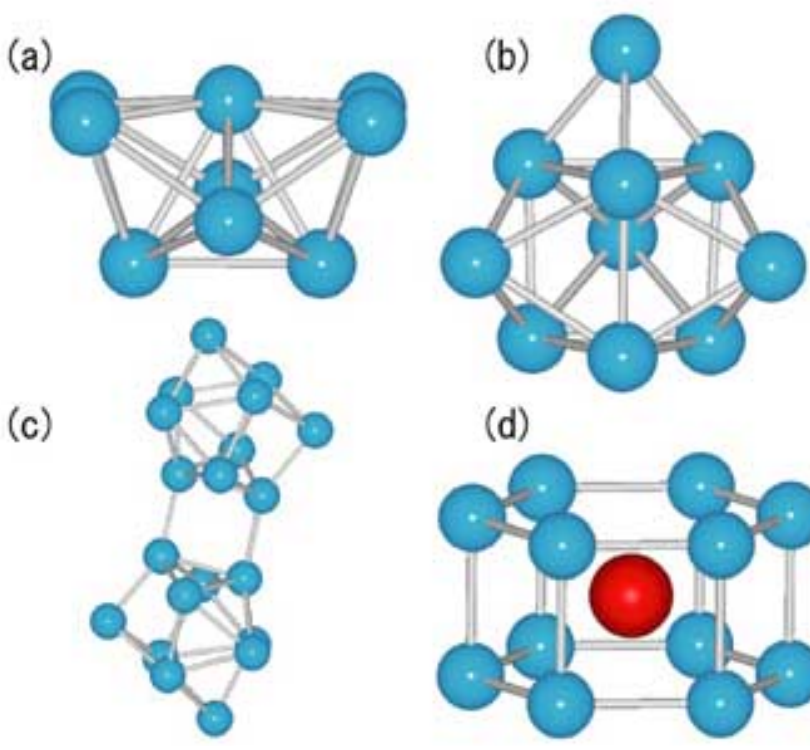

(d)

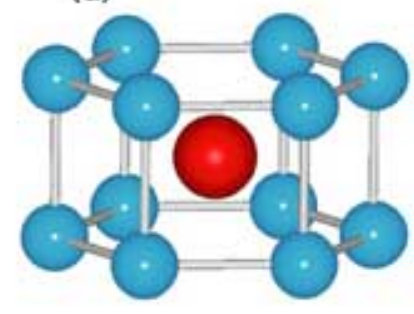

Figure 1. Lowest energy structures of (a) $\mathrm{Si}_{9}$, (b) $\mathrm{Si}_{10}$, (c) prolate structure of $\mathrm{Si}_{20}$ obtained from quantum Monte Carlo method (Mitas et al 2000), and (d) $\mathrm{Si}_{12} \mathrm{~W}$ with $\mathrm{W}$ (black filled circle) at the centre (Hiura et al 2001). 
cage such that there is a tendency for the shrinkage of the cage (figure 2(b)).

Following this result and adopting an atom removal and cage shrinkage approach, novel M encapsulated

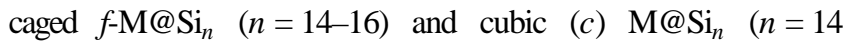
and 15) structures (the notation $\mathrm{M} @ \mathrm{Si}_{n}$ is used to denote caged structures of $n \mathrm{Si}$ atoms around an $\mathrm{M}$ atom without cappings). The shape and size of these clusters depend upon the size of the $\mathrm{M}$ atom which also determines the gap. Also a Frank-Kasper $(F K)$ polyhedral $\mathrm{M} @ \mathrm{Si}_{16}$, $(\mathrm{M}=\mathrm{Ti}$ and $\mathrm{Hf}$ ) cluster has been found to have an exceptionally large gap of about $2.35 \mathrm{eV}$ that makes this cluster attractive for opto-electronic materials. The embedding energy (EE) of the $\mathrm{M}$ atom in the cage is very large $(\approx 12 \mathrm{eV})$ that leads to a strong stability of these clusters. This together with the variable band gap and weak interaction between clusters make this new family of structures, interesting for self-assembled materials. Similar studies have been done on $\mathrm{Ge}$ and $\mathrm{Sn}$ clusters. In particular, doping of divalent $\mathrm{M}$ atoms has led to the finding of icosahedral $(i)$ clusters of $\mathrm{Ge}$ and $\mathrm{Sn}$ for the first time (Kumar and Kawazoe 2002a). In the following, we present a brief review of these results.

\section{Computational approach}

The calculations were performed using $a b$ initio ultrasoft pseudopotential plane wave method (Kresse and Fürthmüller 1996). The exchange-correlation energy was calculated within the spin-polarized generalized gradient approximation (Perdew 1991). A simple cubic supercell with size up to $18 \AA$ was used with periodic boundary

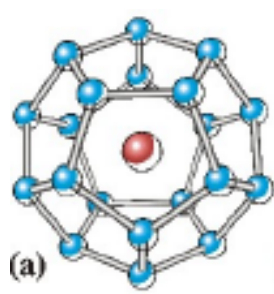

(b)
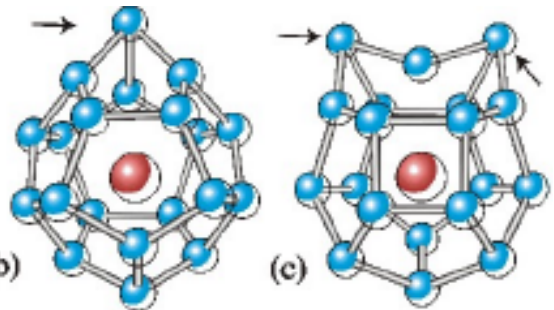

(d)
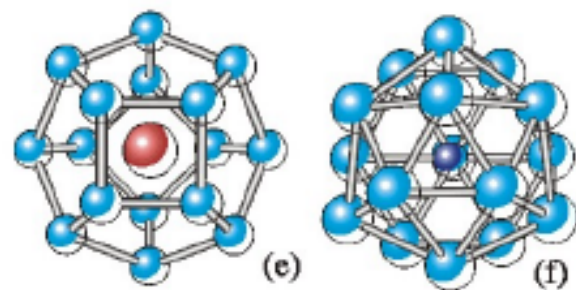

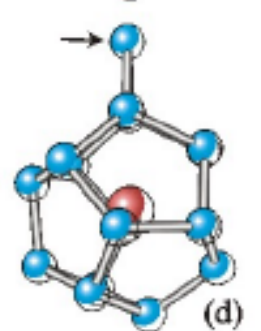

Figure 2. Shrinkage of the $\mathrm{Si}_{20}$ cage. (a) Dodecahedral $\mathrm{Zr}$ encapsulated $\mathrm{Zr} @ \mathrm{Si}_{20},(\mathbf{b})-(\mathbf{e})$ optimized structures of $\mathrm{Zr} @ \mathrm{Si}_{20}$, $\mathrm{Zr} @ \mathrm{Si}_{19}, \mathrm{Zr} @ \mathrm{Si}_{17}$, and silicon fullerene $\mathrm{Zr} @ \mathrm{Si}_{16}$, respectively. Arrows indicate the atoms that were removed. The same structure is obtained for $\mathrm{Hf} @ \mathrm{Si}_{16}$. (f) The Frank-Kasper polyhedral structure of $\mathrm{M} @ \mathrm{Si}_{16}(\mathrm{M}=\mathrm{Ti}$ and $\mathrm{Hf})$. The atom in the cage is M (after Kumar and Kawazoe 2001). conditions and the $\Gamma$ point, for the Brillouin zone integrations. The structures were optimized using the conjugate gradient method. The relaxed structures were found to have zero spin in most cases.

\section{The cage shrinking approach}

$\mathrm{Zr}$ is among the largest size atoms in transition $\mathrm{M}$ series. Its encapsulation in the smallest carbon fullerene cage structure of $\mathrm{Si}_{20}$ (figure 2(a)) led to a $\mathrm{Si}$ atom sticking out (figure 2(b)) upon relaxation. Removal of this capping atom and reoptimization led to a $\mathrm{Zr} @ \mathrm{Si}_{17}$ cage with two $\mathrm{Si}$ atoms sticking out (figure 2(c)). Further removal of the two capping $\mathrm{Si}$ atoms and reoptimizations led to the $\mathrm{Zr} @ \mathrm{Si}_{16}$ cage with a capping by a $\mathrm{Si}$ atom (figure 2(d)). Again removal of this capping atom and reoptimization

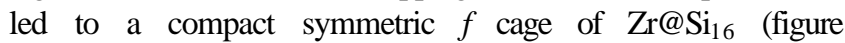
2(e)). This procedure of shrinkage and removal of atoms was continued with smaller $M$ atoms starting with the structure of $f-\mathrm{Zr} @ \mathrm{Si}_{16}$. It further led to the findings of caged $\mathrm{M} @ \mathrm{Si}_{14}$ and $\mathrm{M} @ \mathrm{Si}_{15}$ clusters. The stability of these clusters was further checked by optimizing several other structures. This procedure was repeated for germanium also. Studies on 12-atom cage structures further led to the finding of $i$ isomers.

\section{Results}

The close packed symmetric $f$ cage of $16 \mathrm{Si}$ atoms (figure 2(e)) has eight pentagonal and two square faces as compared to 12 regular pentagonal faces in the dodecahedral $\mathrm{Si}_{20}$ (figure 2(a)). Each $\mathrm{Si}$ atom on the cage is tricoordinated similar to carbon fullerenes. The size of $f-\mathrm{Zr} @ \mathrm{Si}_{16}$ is close to that of $\mathrm{C}_{60}$, but the former is lighter. The $\mathrm{Si}-\mathrm{Si}$ bond lengths are slightly smaller than in bulk $\mathrm{Si}$ and the bonding is covalent. The encapsulation of $\mathrm{M}$ atom leads to a large gain in energy that makes these $\mathrm{M}$ doped clusters to be the most stable $\mathrm{Si}$ clusters known so far. The HOMO-LUMO gaps are also large (table 1) and this should make such clusters very abundant. The energy gain in adding one $\mathrm{Si}$ atom on $f-\mathrm{Zr} @ \mathrm{Si}_{16}$ cage is only $3.211 \mathrm{eV}$ and the $\mathrm{BE}$ of the $\mathrm{Si}_{17} \mathrm{Zr}$ structure (figure 3(d)), $4 \cdot 109 \mathrm{eV} /$ atom. This is lower than the value of $4.162 \mathrm{eV} /$ atom for the $f-\mathrm{Zr} @ \mathrm{Si}_{16}$ cage. Therefore, $f-\mathrm{Zr}_{\mathrm{S}} \mathrm{Si}_{16}$ shows

Table 1. BEs (eV/atom), EEs (eV), and HOMO-LUMO gaps $(\mathrm{eV})$ of the $f$ and $F K$ isomers of $\mathrm{M} @ \mathrm{Si}_{16}$ clusters.

\begin{tabular}{lccc}
\hline Cluster & BE & EE & Gap \\
\hline$F K-\mathrm{Ti} @ \mathrm{Si}_{16}$ & $4 \cdot 135$ & $11 \cdot 269$ & $2 \cdot 358$ \\
$f-\mathrm{Ti} @ \mathrm{Si}_{16}$ & $4 \cdot 089$ & $12 \cdot 733$ & 1.495 \\
$f-\mathrm{Zr} \mathrm{Si}_{16}$ & $4 \cdot 162$ & 13.965 & $1 \cdot 580$ \\
$F K-\mathrm{Zr} @ \mathrm{Si}_{16}$ & $4 \cdot 141$ & $11 \cdot 814$ & $2 \cdot 448$ \\
$f-\mathrm{Hf} @ \mathrm{Si}_{16}$ & $4 \cdot 175$ & $14 \cdot 176$ & $1 \cdot 576$ \\
$F K-\mathrm{Hf} @ \mathrm{Si}_{16}$ & $4 \cdot 171$ & $12 \cdot 399$ & $2 \cdot 352$ \\
\hline
\end{tabular}



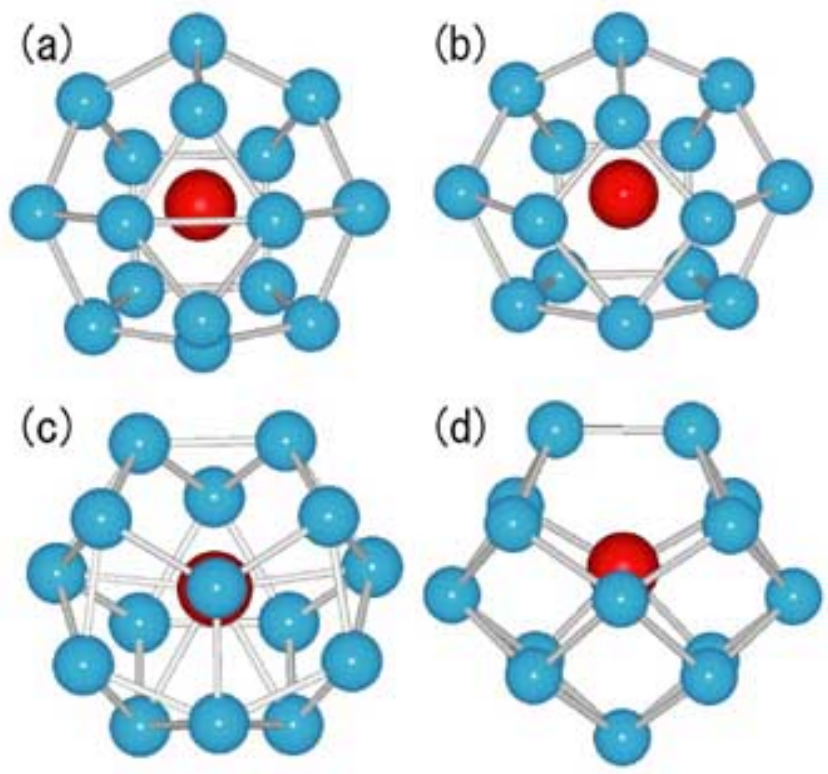

Figure 3. (a) Optimized structure of $f-\mathrm{M} @ \mathrm{Si}_{16}$ with $\mathrm{M}=\mathrm{Cr}$. There is a shrinkage of the silicon cage, (b) the capping silicon atom in (a) is removed and the structure, reoptimized to obtain the $f$-Cr@Si $i_{15}$ isomer, (c) the $c$ isomer of $\mathrm{Cr} @ \mathrm{Si}_{15}$ that has the lowest energy, (d) lowest energy isomer of $\mathrm{M} @ \mathrm{Si}_{15}, \mathrm{M}=\mathrm{Ti}$, $\mathrm{Zr}$, Hf, Ru, and Os. Structures of $\mathrm{Si}_{16} \mathrm{M}$ and $\mathrm{M} @ \mathrm{Si}_{15}$ for $\mathrm{M}=\mathrm{Mo}$ and $\mathrm{W}$ are similar (after Kumar and Kawazoe 2002b).

magic behaviour. Similar results were obtained for the isoelectronic $\mathrm{Hf} @ \mathrm{Si}_{16}$. However, encapsulation of $\mathrm{Ti}$ in the $f$ cage does not lead to closest packing due to its smaller size and an $F K$ polyhedron (figure 2(f)) was found to be $0.781 \mathrm{eV}$ lower in energy. It has tetrahedral symmetry with four connected capped hexagons of $\mathrm{Si}$. The Ti-Si bond lengths in this structure are slightly reduced and it enhances interaction of $\mathrm{Ti}$ with the $\mathrm{Si}$ cage. The Si-Si bonds are, however, elongated (2.37-2.66 $\AA$ ) and have a mixed covalent-metallic character. This isomer has an exceptionally large gap of $2.358 \mathrm{eV}$ that lies in the visible range. It is likely that the actual gap would be significantly higher as the approximate density functional methods tend to underestimate the gap. Similar calculations were performed for $\mathrm{M}=\mathrm{Hf}$ and $\mathrm{Zr}$. The isoelectronic $F K-\mathrm{Hf} @ \mathrm{Si}_{16}$ was found to be nearly degenerate with the $f$ isomer, but its HOMO-LUMO gap is as large $(2.352 \mathrm{eV})$ as for $\mathrm{M}=\mathrm{Ti}$. However, $F K-\mathrm{Zr} @ \mathrm{Si}_{16}$ lies $0.355 \mathrm{eV}$ higher in energy than the $f$ isomer. These results suggested that both $\mathrm{Ti}$ and $\mathrm{Hf}$ encapsulated $\mathrm{Si}$ clusters with $F K$ polyhedron structure should be strongly abundant and that $\mathrm{Hf}$ doping is unique to produce two different stable degenerate isomers with different bonding natures and significantly different band gaps. These are the only cages with 16 silicon atoms that are formed with even electron transition $M$ atoms. In the case of other $\mathrm{M}$ atoms, 16 silicon atom clusters are possible but either these are not of lowest energy or there are distortions or the cage shrinks with capping of some $\mathrm{Si}$ atom(s). Interaction between two $f$ clusters has been found to be relatively weak with $1.345 \mathrm{eV} \mathrm{BE}$ and a reduction in the gap. However, for $F K$ isomers, this interaction is very weak $(0.048 \mathrm{eV})$ and the gap remains large, making these species attractive for self-assembly.

Encapsulation of smaller $\mathrm{M}$ atoms such as $\mathrm{Cr}$, Mo, and $\mathrm{W}$ led to the shrinkage of $f-\mathrm{M} @ \mathrm{Si}_{16}$ to $\mathrm{M} @ \mathrm{Si}_{15}$ cage with a capping atom (Kumar and Kawazoe 2002b) as shown in figure 3(a). Removal of the capping atom and subsequent reoptimization led to a stable $f-\mathrm{M} @ \mathrm{Si}_{15}$ cage (figure 2(b)). The HOMO-LUMO gap is small $(0.808 \mathrm{eV})$ and the $\mathrm{BE}, 3.883 \mathrm{eV} /$ atom. The $\mathrm{EE}$ of $\mathrm{Cr}$ in the cage is large $(8.936 \mathrm{eV})$ but significantly lower than $\approx 14 \mathrm{eV}$ obtained for $\mathrm{Zr}$ and $\mathrm{Hf}$ (table 1) due to complete quenching of the magnetic moment of $\mathrm{Cr}$. The $\mathrm{Si}-\mathrm{Si}$ bond lengths are in the range of $2.27-2.34 \AA$ indicating covalent bonding in this isomer. A few bonds are, however, elongated with values of 2.47 and $2.72 \AA$. The $\mathrm{Cr}-\mathrm{Si}$ bond lengths lie in the range of $2.60-2.96 \AA$. These values are more than the sum of the covalent radii, 1.18 and 1.36 of $\mathrm{Si}$ and $\mathrm{Cr}$ atoms, respectively. Therefore, $\mathrm{Cr}$ is not optimally bonded in this $f$ silicon cage.

Another 15-atom Si cage was attempted from a body centred cubic structure by placing one more $\mathrm{Si}$ atom on a face of the cube with $\mathrm{M}$ at the centre. Optimization with $\mathrm{M}=\mathrm{Cr}$ led to the structure shown in figure 3(c). It lies $1.071 \mathrm{eV}$ lower in energy than the $f$ isomer and has a large $(1.537 \mathrm{eV})$ HOMO-LUMO gap suggesting its strong stability. This structure is completely different from the $f$ isomer. The mean coordination of $\mathrm{Si}$ atoms on this cage is 4 . The higher coordination of $\mathrm{Si}$ atoms in this structure leads to slightly elongated $\mathrm{Si}-\mathrm{Si}$ bonds (2.36$2.57 \AA$ ). This range is more than the value of $2.35 \AA$ for the covalently bonded bulk silicon and suggests development of some metallic character in this isomer as one would also expect from a higher coordination of $\mathrm{Si}$ atoms. The $\mathrm{M}-\mathrm{Si}$ bond is, however, slightly shorter (2.54-2.83 $\AA$ ) than in the $f$ isomer with majority of bonds having the lower value. This leads to a better M-Si interaction in this structure. The valence states of $\mathrm{Cr}$ atom lie near the HOMO of the $\mathrm{Si}$ cage and interact covalently with the latter and the nearby states. This leads to a lowering of the energy of the $\mathrm{Si}$ cage states in a range of about $2 \mathrm{eV}$ below the HOMO but the deeper states are only weakly perturbed. The same holds true also for other $\mathrm{M}$ atoms.

Similar calculations on $\mathrm{M} @ \mathrm{Si}_{16}$ with $\mathrm{M}=\mathrm{Mo}$ and $\mathrm{W}$ showed the capped caged structure of figure $3(\mathrm{a})$ to be the lowest in energy similar to $\mathrm{Cr}$. Optimizations for $\mathrm{M} @ \mathrm{Si}_{15}$ with $\mathrm{M}=\mathrm{Mo}$ and $\mathrm{W}$, respectively, showed an increase in the gap to 1.659 and $1.747 \mathrm{eV}$ in the $c$ derived structure. Therefore, for $\mathrm{M} @ \mathrm{Si}_{15}(\mathrm{M}=\mathrm{Cr}, \mathrm{Mo}$, and $\mathrm{W})$, 
the isomer obtained from a $c$ structure has the lowest energy and large HOMO-LUMO gap. The bonding properties of these clusters with isoelectronic dopings are similar. The different growth modes of 15 and $16 \mathrm{Si}$ atom clusters lead to their simultaneous large abundances as observed (Beck 1989). Strikingly, these are the only two clusters with high intensities in experiments and beyond this size there is very low intensity of other clusters. These results show the importance of the $\mathrm{M} @ \mathrm{Si}_{15}$ cage for these $\mathrm{M}$ atoms and suggest that the only other significantly stable cluster with a capping is $\mathrm{Si}_{16} \mathrm{M}$ that has large gap. Therefore, it is highly likely that such caged clusters can be uniquely produced for developing cluster assembled materials with desired properties as the shape, size, and band gap can be varied with a suitable choice of M. Further calculations (Kumar and Kawazoe 2002b) on $\mathrm{M}=\mathrm{Ti}, \mathrm{Zr}, \mathrm{Hf}, \mathrm{Ru}$, and $\mathrm{Os}$ for the 15-atom silicon cage give a slightly different $c$-M@ $\mathrm{Si}_{15}$ structure with large BEs and about 1.2 eV HOMO-LUMO gaps.

Studies (Kumar and Kawazoe 2001) with further smaller $\mathrm{M}$ atoms, $\mathrm{Fe}, \mathrm{Ru}$, and $\mathrm{Os}$ showed the $f-\mathrm{M} @ \mathrm{Si}_{16}$ cage to shrink to $\mathrm{M} @ \mathrm{Si}_{14}$ with two $\mathrm{Si}$ atoms capping (figure 4(a)). Removal of these two capping atoms and subsequent optimizations led to a $f-\mathrm{M} @ \mathrm{Si}_{14}$ cage (figure 4(b)) which is better described as a capped decahedron (d). It was, however, found that a body centred cubic structure (figure 3(f)) had lower energy and larger HOMO-LUMO gap. But in the case of $M=R u$ and Os,
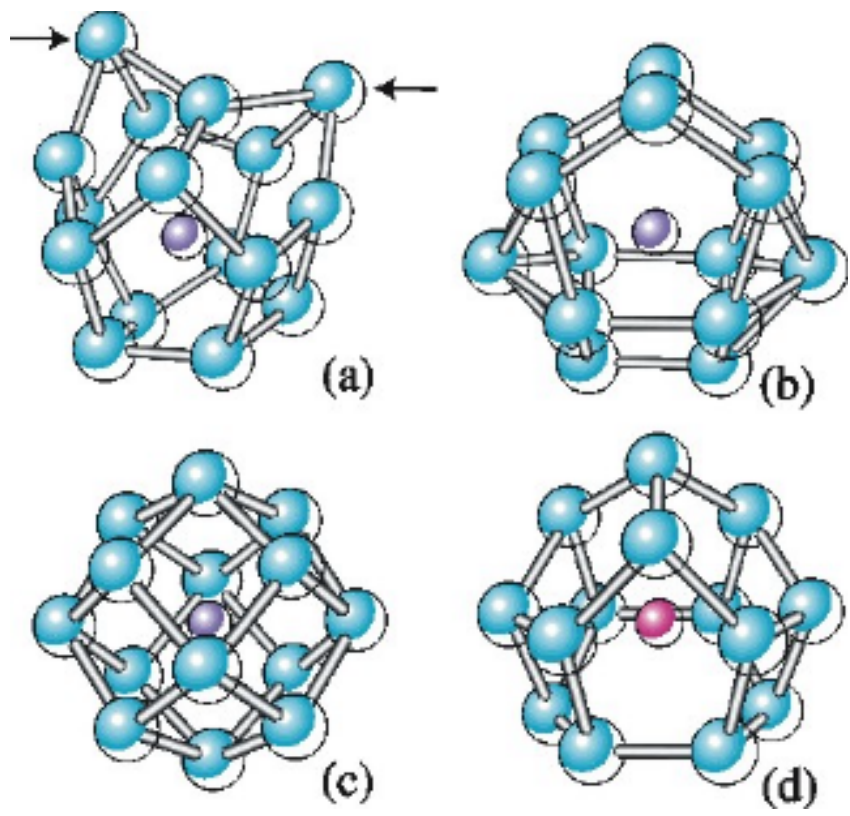

Figure 4. Shrinkage of $f$-Fe@ $\mathrm{Si}_{16}$ structure: (a) optimized $\mathrm{Si}_{16} \mathrm{Fe}$ with two $\mathrm{Si}$ atoms capping (marked with arrows), (b) $d$ $\mathrm{M} @ \mathrm{Si}_{14}, \mathrm{M}=\mathrm{Fe}$ and $\mathrm{Ru}$, (c) $c$-Fe@Si $\mathrm{Si}_{14}$, and (d) $f$-Os@ $\mathrm{Si}_{14}$. The $c$ structures of $\mathrm{Ru}$ and Os are similar to (c) (after Kumar and Kawazoe 2001). both the $f$ and $c$ isomers are nearly degenerate. The BE, $\mathrm{EE}$ and the HOMO-LUMO gaps of these clusters are given in table 2. Though, $\mathrm{M} @ \mathrm{Si}_{15}$ structure has also been obtained for $\mathrm{M}=\mathrm{Ru}$ and $\mathrm{Os}$, it is not close packed. These calculations suggested the 14-atom $\mathrm{Si}$ cage to be optimal for $\mathrm{M}=\mathrm{Fe}, \mathrm{Ru}$, and Os. Calculations with $\mathrm{M}=\mathrm{Ni}, \mathrm{Pd}$, and $\mathrm{Pt}$ also gave cubic structure to be of lowest energy, but there is a magnetic moment of $2 \mu_{\mathrm{B}}$ and the HOMOLUMO gap is small $(<0.5 \mathrm{eV})$. Therefore, these clusters are unlikely to be abundant.

Similar studies (Kumar and Kawazoe 2002c) have been carried out on germanium clusters. In this case the $f$-M@ $\mathrm{Ge}_{16}$ structure transforms into the $F K$ polyhedron which again has a large HOMO-LUMO gap of about $2 \mathrm{eV}$ similar to the $\mathrm{Si}$ based clusters. The 14- and 15atom clusters have a tendency to prefer capped decahedral structures. It is due to the weaker covalent character of Ge. The lowest energy structures of 14,15 , and $16 \mathrm{Ge}$ atom cages are shown in figure 5. Again there is a large gain in the binding energy as compared to the elemental Ge clusters.

Further studies have been carried out on 12 atom cages with $\mathrm{M}$ encapsulation. For $\mathrm{Si}_{12} \mathrm{M}, \mathrm{M}=\mathrm{Cr}$, $\mathrm{Mo}$, and $\mathrm{W}$, a $h$ prism (figure $1(\mathrm{~d})$ ) has the lowest energy. The $\mathrm{BE}$ and gaps are 3.84, 4.02, and $4.18 \mathrm{eV}$ and 0.85, 0.90, and $1.34 \mathrm{eV}$, respectively. These gaps are lower than the values obtained for the 15- and 16-atom $\mathrm{Si}$ cages except for $\mathrm{Si}_{12} \mathrm{~W}$, but the $\mathrm{BE}$ of the 15- and 16-atom cages are higher than the corresponding value for the $\mathrm{Si}_{12} \mathrm{M}$ cluster. Therefore, 15- and 16-atom cages are more stable than $\mathrm{Si}_{12} \mathrm{M}$ in agreement with the finding of the laser evaporation experiments. As the sizes of these $\mathrm{M}$ atoms are significantly larger than that of $\mathrm{Si}$, these cannot form close packed structures with $12 \mathrm{Si}$ atoms. This is, however, possible with smaller $\mathrm{M}$ atoms. Further studies (Kumar and Kawazoe, to be published) of $\mathrm{H}$ interaction with these clusters show weak bonding in agreement with the absence of hydrogenated species of $\mathrm{Si}_{12} \mathrm{M}$. Calculations (Kumar and Kawazoe 2002a; and to be published) on several other $\mathrm{M}$ atoms led to the finding of $i$ clusters of $\mathrm{Ge}$ and $\mathrm{Sn}$ with large gaps by doping divalent $\mathrm{M}$ atoms such as $\mathrm{Be}, \mathrm{Zn}, \mathrm{Mg}$, and $\mathrm{Ca}$.

Table 2. BEs (eV/atom), EEs (eV), HOMO-LUMO gaps $(\mathrm{eV})$ of low lying isomers of selected clusters.

\begin{tabular}{lcrc}
\hline Cluster & BE & \multicolumn{1}{c}{ EE } & Gap \\
\hline$f-\mathrm{Si}_{16} \mathrm{Fe}$ & $4 \cdot 010$ & $9 \cdot 426$ & 1.294 \\
$f-\mathrm{Si}_{16} \mathrm{Ru}$ & $4 \cdot 188$ & $12 \cdot 445$ & 1.230 \\
$f-\mathrm{Si}_{16} \mathrm{Os}$ & $4 \cdot 252$ & 13.551 & 1.246 \\
$c-\mathrm{Fe} @ \mathrm{Si}_{14}$ & $4 \cdot 018$ & $8 \cdot 404$ & 1.162 \\
$c-\mathrm{Ru} @ \mathrm{Si}_{14}$ & $4 \cdot 194$ & $11 \cdot 136$ & 1.409 \\
$f-\mathrm{Ru} @ \mathrm{Si}_{14}$ & $4 \cdot 184$ & 12.939 & 1.488 \\
$c-\mathrm{Os} @ \mathrm{Si}_{14}$ & $4 \cdot 259$ & $12 \cdot 111$ & 1.453 \\
$f-\mathrm{Os} @ \mathrm{Si}_{14}$ & $4 \cdot 258$ & 14.024 & 1.576 \\
\hline
\end{tabular}



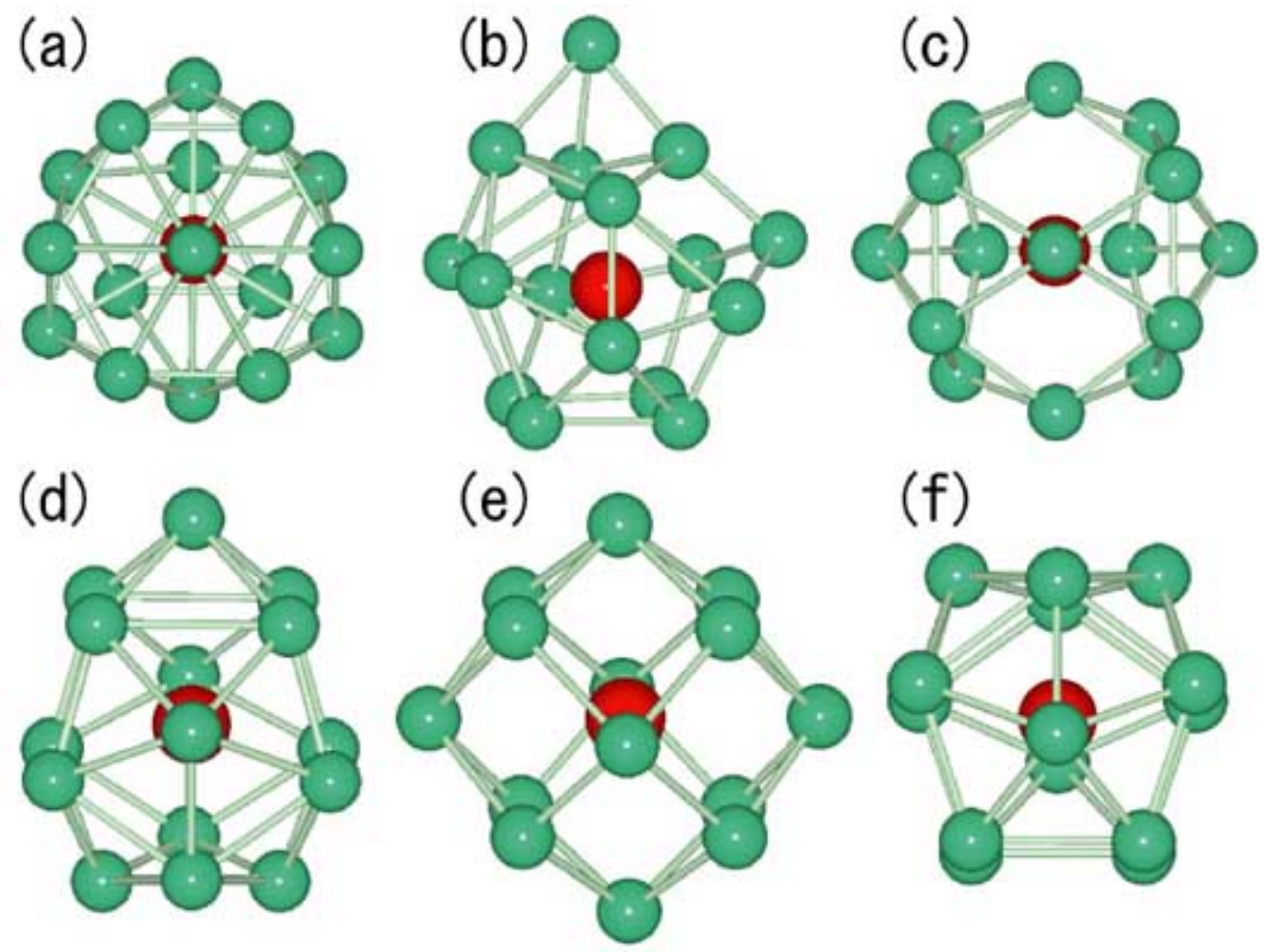

Figure 5. Optimized structure of $\mathrm{M} @ \mathrm{Ge}_{n}$ clusters. (a) $\mathrm{FK}$ polyhedron for $n=16$ and $\mathrm{M}=\mathrm{Ti}$, $\mathrm{Hf}$, and $\mathrm{Zr}$, (b) capped decahedral isomer for $n=16$ and $\mathrm{M}=\mathrm{Cr}$, Mo, and $\mathrm{W},(\mathbf{c})$ and (d) are two closely lying decahedral isomers for $n=15$, (e) and (f) are cubic and decahedral isomers for $n=14$ (after Kumar and Kawazoe 2002c).

\section{Summary}

In summary, we have presented a brief review of the recently found transition $\mathrm{M}$ atom encapsulated silicon and germanium caged clusters from computer experiments based on an ab initio method. These form 14-, 15-, and 16-atom cages with $\mathrm{M}$ at the centre, depending upon the size of the $M$ atom. The shape and the HOMOLUMO gap depend upon M. Most notable among these are the fullerene-like, cubic, and Frank-Kasper polyhedral structures. Interaction between the fullerene-like cages is relatively weak while interaction between the Frank-Kasper polyhedral clusters is van der Waals type. Further icosahedral clusters of $\mathrm{Ge}$ and $\mathrm{Sn}$ have been obtained with divalent $\mathrm{M}$ atom encapsulation. These results show that one $\mathrm{M}$ atom changes the properties of elemental $\mathrm{Si}$ and $\mathrm{Ge}$ clusters drastically. It has opened up new avenues for the development of elements of nanodevices. Also these results can shed new light on the understanding of metal-semiconductor interfaces as well as the development of nanowires for which metal particles are used as catalyst. The strong stability of these clusters results from strong $\mathrm{M}-\mathrm{Si}$ interactions that also fix the size of the cage. It is likely that these clusters can be pro- duced exclusively in large quantities. The HOMOLUMO gaps in these clusters are large and in particular for the $F K$ isomer, these lie in the visible range. This is attractive for opto-electronic applications.

\section{Acknowledgements}

This work has been done in cooperation with Prof. Y Kawazoe. I gratefully acknowledge the kind hospitality at the Institute for Materials Research and the support of the staff of the Centre for Computational Materials Science of IMR-Tohoku University, for the use of SR8000/ H64 supercomputer facilities.

\section{References}

Beck S M 1989 J. Chem. Phys. 906306

Hiura H, Miyazaki T and Kanayama T 2001 Phys. Rev. Lett. 86 1733

Ho K-M, Shvartsburg A A, Pan B, Lu Z Y, Wang C Z, Wacker J G, Fye J L and Jarrold M F 1998 Nature 392582

Jackson K and Nellermoe B 1996 Chem. Phys. Lett. 254249

Jarrold M F and Bower J E 1988 J. Phys. Chem. 925702 
Kresse G and Furthmüller G 1996 Phys. Rev. B55 11169 Kumar V, Esfarjani K and Kawazoe Y 2002 in Clusters and nanomaterials, Springer Series in Cluster Physics (eds) $\mathrm{Y}$ Kawazoe et al (Heidelberg: Springer Verlag) p. 9

Kumar V and Kawazoe Y 2001 Phys. Rev. Lett. 87045503

Kumar V and Kawazoe Y 2002a Appl. Phys. Lett. 80859

Kumar V and Kawazoe Y 2002b Phys. Rev. B65 073404
Kumar V and Kawazoe Y 2002c Phys. Rev. Lett. 88235504

Mitas L, Grossman J C, Stich I and Tobik J 2000 Phys. Rev. Lett. 841479

Perdew J P 1991 in Electronic structure of solids '91 (eds) P Ziesche and H Eschrig (Berlin: Akademie Verlag)

Röthlisberger U, Andreoni W and Parrinello M 1994 Phys. Rev. Lett. 72665 